\title{
Early neonatal sepsis and meningitis caused by Elizabethkingia meningoseptica in Saudi Arabia
}

Ashwaq I. Barnawi, MBBS, MD, Faisal N. Kordy, MBBS, MD, Ohoud K. Almuwallad, MBBS, MD, Khloud A. Kassarah, MBBS, MD.

\begin{abstract}

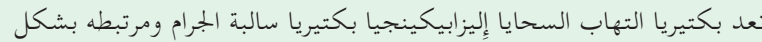

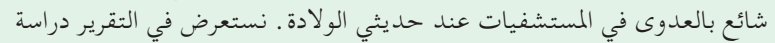

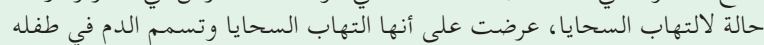

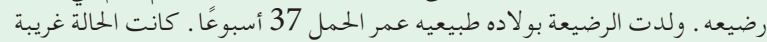

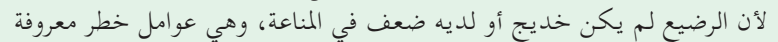

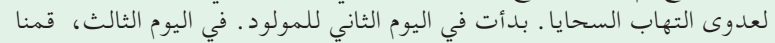

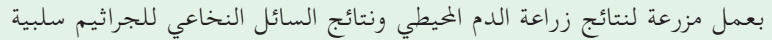

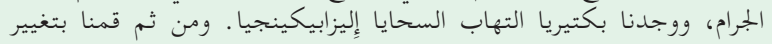

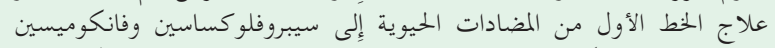

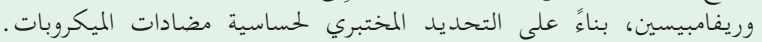

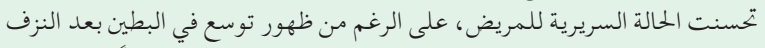

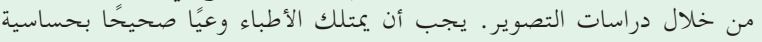

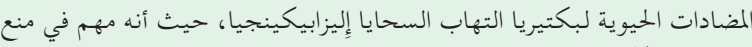

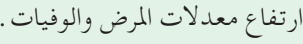

Elizabethkingia meningoseptica (E. meningoseptica) are Gram-negative bacteria commonly associated with nosocomial infections in neonates. This is a case study of E. meningoseptica, presented as meningitis and sepsis in a term baby. The female infant was born by vaginal delivery at 37 weeks gestational age. The case was peculiar because the baby was neither premature nor immuno-compromised, which are known risk factors for E. meningoseptica infection. The onset began on the second day of the neonate's life. On day 3, peripheral blood culture and cerebrospinal fluid findings isolated a gram-negative bacteria identified as E. meningoseptica. The first-line antibiotics therapy was changed to ciprofloxacin, vancomycin, and rifampicin, based on the laboratory determination of antimicrobial sensitivity. The patient's clinical condition improved, although post hemorrhagic ventricular dilatation was revealed by imaging studies. Clinicians should possess proper awareness of the antibiotic sensitivity of $E$. meningoseptica, as it is important in preventing high rates of morbidity and mortality.

Keywords: Elizabethkingia meningoseptica, meningitis, neonate, bacteremia

Saudi Med J 2020; Vol. 41 (7): 753-756 doi: 10.15537/smj.2020.7.25720
From the Department of Pediatrics (Barnawi), Madina Maternity \& Children's Hospital; from the Department of Pediatrics (Kordy), from the Department of Neonatal Intensive Care Development (Almuwallad); and from the Department of General Pediatric Development (Kassarah), Maternity and Child Hospital, Ministry of Health, King Abdullah Medical City, Al-Madina Al-Munawara, Kingdom of Saudi Arabia.

Received 13th November 2019. Accepted 21st April 2020.

Address correspondence and reprint request to: Dr. Ashwaq I. Barnawi, Department of Pediatrics, Madina Maternity \& Children's Hospital, Al-Madina Al-Munawara, Kingdom of Saudi Arabia. E-mail: ha_modi1430@hotmail.com

ORCID ID: https://orcid.org/0000-0002-4109-1143

E lizabethkingia meningoseptica (formerly referred to as Flavobacterium meningosepticum, Chryseobacterium meningosepticum), is a ubiquitous, glucose nonfermenting, non-motile, catalase, oxidase positive, and aerobic gram-negative bacillus, commonly found in natural environments such as fresh or salt water, soil and also in hospital environments via contaminated medical equipment, particularly in neonatal wards. ${ }^{1,2}$ It was first reported by bacteriologist Elizabeth O. King in 1959, at the Centers for Disease Control in Atlanta, Georgia, United States of America. ${ }^{3}$ It was then named after the original discoverer. Elizabethkingia meningoseptica (E. meningoseptica) has an unusual responsiveness to antibiotics, usually resistant to majority of antibiotics commonly used in treating gram-negative bacteria infections, but highly susceptible to antibiotics such as clindamycin, cotrimoxazole, and erythromycin, usually used to treat gram-positive bacterial infections which may lead to inappropriate antibiotic use. ${ }^{3}$

Elizabethkingia meningoseptica although rare has been known to cause infections in neonates at neonatal

Disclosure. Authors have no conflict of interests, and the work was not supported or funded by any drug company. 
intensive care units, commonly leading to meningitis and sepsis. ${ }^{4,5}$ In around half of the cases, the organism is responsible for severe sequalae, such as brain abscess, developmental delay, deafness, and hydrocephalus. ${ }^{4,6}$ Reported below is a case of early neonatal sepsis and meningitis caused by E. meningoseptica. In this case, the infection was acquired off-site.

Case Report. This was a case of a female baby born by vaginal delivery at 37 weeks gestation weighing 2,600 grams. The mother was a primigravida with no known risk factors. She presented to the emergency department on the second day of the neonate's life due to tachypnea since birth, and with a fever of $38.7^{\circ} \mathrm{C}\left(101.6^{\circ} \mathrm{F}\right)$ for one day, and a decreased level of consciousness without abnormal movements.

Clinical findings \& diagnostic assessment. A blood sample was taken and revealed anemia and thrombocytopenia platelet count $81 \times 10^{3} / \mathrm{uL}$. There was a derangement of coagulation profile with partial thromboplastin time (PTT) 53s, and C-reactive protein was $91 \mathrm{mg} / \mathrm{dL}$. Persistent metabolic acidosis with high lactate $(7 \mathrm{mmol} / \mathrm{L})$ was noted. She was admitted to the neonatal intensive care unit (NICU) with tachypnea since birth, high grade fever $38.7^{\circ} \mathrm{C}\left(101.6^{\circ} \mathrm{F}\right)$ Decrease level of consciousness, with no abnormal movements and in compensated shock state, intubated, ventilated, brain ultrasound showed prominent bilateral ventricles and a lumbar puncture was performed. Cerebrospinal fluid analysis showed: glucose $(2.9 \mathrm{mg} / \mathrm{dL})$; proteins $(159 \mathrm{mg} / \mathrm{dL})$, leucocyte $\left(95 \mathrm{cell} / \mathrm{mm}^{3}\right), 0 \%$ neutrophils, and $100 \%$ lymphocyte. She was admitted and empirical treatment with intravenous double-dose ampicillin and cefotaxime was initiated. Cranial ultrasound showed bilateral grade-1 intraventricular hemorrhage. She developed convulsions which were controlled through intravenous phenobarbitone. On the third day, peripheral blood culture and cerebrospinal fluid microbiology isolated a gram-negative bacteria identified as Chryseobacterium- E. meningoseptica. The isolated gram-negative, non-fermenting, non-motile, oxidase-positive organism grew well on chocolate agar and blood agar. Colonies had pale yellow coloration and were not readily evident at 24 hours (Table 1).

Therapeutic intervention. The patient was admitted and empirical treatment with intravenous double-dose ampicillin and cefotaxime was initiated. The organism was found to be sensitive to ciprofloxacin and levofloxacin, with intermediate sensitivity to tigecycline, and it was resistant to colistin, trimethoprimsulfamethoxazole, third-generation cephalosporins and carbapenems. The initial combination of antibiotics therapy was changed to ciprofloxacin vancomycin for a total of 6 weeks and rifampicin added for the initial

Table 1 - Clinical and outcome description with specific dates and times of therapeutic intervention.

\begin{tabular}{|c|c|c|c|}
\hline Dates & \multicolumn{3}{|c|}{ Relevant past medical history and interventions } \\
\hline \multirow[t]{2}{*}{15 March 2019} & \multicolumn{3}{|c|}{ Newborn female infant, 37 weeks, spontaneous vaginal delivery after uncomplicated pregnancy, with birth weight $2600 \mathrm{~g}$. } \\
\hline & Summaries from initial and follow-up visits & Diagnostic testing & Interventions \\
\hline 16-17 March 2019 & $\begin{array}{l}\text { Presentation with tachypnea since birth, } \\
\text { high grade fever } 38.7^{\circ} \mathrm{C}\left(101.6^{\circ} \mathrm{F}\right) \\
\text { Decrease level of consciousness, with no } \\
\text { abnormal movements } \\
\text { Compensated shock status, with persistent } \\
\text { metabolic acidosis }\end{array}$ & $\begin{array}{l}\text { Complete blood count, C-reactive } \\
\text { protein, lactate, coagulation } \\
\text { profile, blood gas, blood culture } \\
\text { and serum electrolytes, chest x-ray. } \\
\text { Amplitude cerebral function } \\
\text { monitoring cranial ultrasound. }\end{array}$ & $\begin{array}{l}\text { Intubated ventilated lumber } \\
\text { puncture started on first line } \\
\text { antibiotics. } \\
\text { Anti-epileptic medication. }\end{array}$ \\
\hline 18 March - 10 April 2019 & $\begin{array}{l}\text { Isolated organism from culture of blood } \\
\text { and cerebrospinal fluid with antimicrobial } \\
\text { sensitivity } \\
\text { Consciousness, shock and acidosis }\end{array}$ & $\begin{array}{l}\text { Repeated blood culture. } \\
\text { Repeated CSF culture, computed } \\
\text { tomography. }\end{array}$ & $\begin{array}{l}\text { Consultation of infectious } \\
\text { department. } \\
\text { Change of antimicrobial } \\
\text { therapy according to } \\
\text { sensitivity to complete total } \\
6 \text { weeks. } \\
\text { Successfully extubated. }\end{array}$ \\
\hline 11 April - 5 May 2019 & & $\begin{array}{l}\text { Brain magnetic resonance. } \\
\text { Follow up brain magnetic } \\
\text { resonance }\end{array}$ & Consultation of neurosurgery. \\
\hline 8 May 2019 & $\begin{array}{l}\text { Clinically stable, breathing room air, } \\
\text { satisfactory feeding, unremarkable } \\
\text { Neurological exam }\end{array}$ & $\begin{array}{l}\text { Magnetic resonance imaging } \\
\text { follow up }\end{array}$ & $\begin{array}{l}\text { Discharge with follow up out- } \\
\text { patient clinic. }\end{array}$ \\
\hline
\end{tabular}




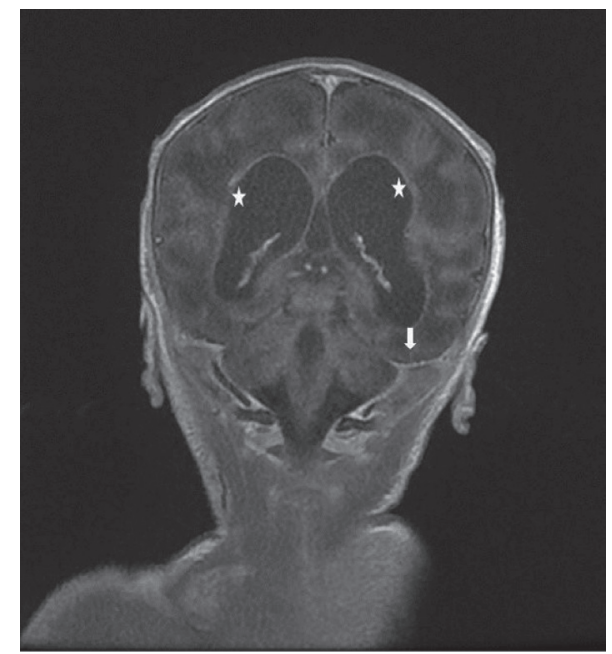

Figure 1 - Magnetic resonance imaging coronal $\mathrm{T} 1$ post contrast image showing basal meningeal enhancement (arrow) and ependymal enhancement of the lateral ventricular wall (star).

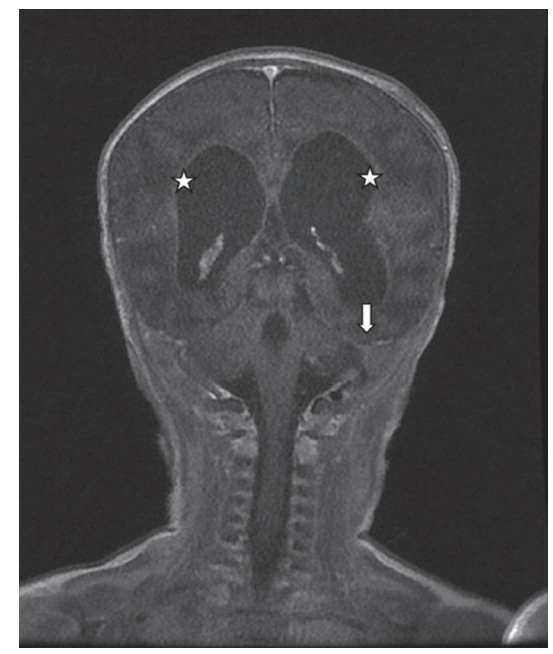

Figure 2 - Follow up MRI cronal T1 post contrast image showing improvement of the basal meningeal enhancement and ependymal enhancement of the lateralventicular wall (star).
2 weeks of therapy. As the patient was born off-site, it was determined unnecessary to take further cultures or swabs to identify the source of infection.

Follow-up and outcomes. A blood culture repeated 48 hours after the start of treatment yielded the same organism with the same sensitivities. Subsequent blood culture at 96 hours was sterile. Magnetic resonance imaging of the brain showed multiple sub-ependymal foci of hemorrhage, ischemic insults, sequel cystic encephalomalacia in periventricular areas, and improvements of basal leptomeningeal enhancement. She was discharged. She was able to breathe without assistance and had satisfactory full oral feeding. Brain magnetic resonance imaging follow-up at the time of discharge showed post hemorrhagic ventricular dilatation (Figures $1 \& 2$ ).

Discussion. Elizabethkingia meningoseptica bacterial infections although rare, have been found to be mostly nosocomial in nature, affecting immunocompromised hosts and preterm neonates. ${ }^{3,6}$ It has generally been reported as a causative agent in outbreaks of meningitis in neonatal intensive care units (ICUs). ${ }^{7}$ Our case is unique in that the patient was infected by E. meningoseptica with symptoms appearing in the first 2 days of life. The baby was not premature and was not immuno-compromised, which are known risk factors for acquiring the infection. A case of neonatal infections caused by E. meningoseptica in Romania reported that the onset of symptoms (fever) began 5 days after birth. ${ }^{1}$ A case of E. meningoseptica bacteremia in a neonate where nosocomial septicemia was diagnosed in a 10-day-old neonate born through normal vaginal delivery, has also been reported. ${ }^{3}$ Elizabethkingia meningoseptica infection in adults shows a tendency to infect immuno-compromised hosts with indwelling devices, and it is nosocomial. ${ }^{8}$ Finally, we should be aware that E. meningoseptica infections can be fatal, and with its peculiar antibiotic profile, the right choice of initial antibiotic therapy is somewhat difficult for clinicians. The possibility of administering inadequate antibiotic therapy is increased due to variance in the results of susceptibility testing when different methods are used. Broth micro dilution is the preferred method for susceptibility testing, as disc diffusion methods may be unreliable. ${ }^{8}$ In other to reduce high rates of morbidity and mortality, E. meningosepticum infection should always be suspected by clinicians when gram-negative bacilli are detected on gram staining, and they should be conversant with its antibiotic susceptibility profile.

In conclusion, E. meningoseptica is a rare but lethal organism. The detection of gram-negative bacilli on gram staining or in culture, should always cause clinicians to suspect E. meningosepticum infection in neonates, even in term babies without obvious risk factors. Proper awareness of antibiotics is necessary to reduce high rates of morbidity and mortality.

Acknowledgment. The authors gratefully acknowledge Dr. Howaida from the Department of Radiology, Maternity and children hospital, Kingdom of Saudi Arabia, for providing a review of magnetic resonance brain imaging and attesting to its clarity and correctness. Also we would like to thank Scribendi (www.scribendi.com) for English language editing. 


\section{References}

1. Arbune M, Fotea S, Nechita A, Stefanescu V. Emerging infection with Elizabethkingia Meningoseptica in Neonate. A case report. J Crit Care Med (Targu Mures) 2018; 4: 96-100.

2. Jean SS, Hsieh TC, Ning YZ, Hsueh PR. Role of vancomycin in the treatment of bacteraemia and meningitis caused by Elizabethkingia meningoseptica. Int J Antimicrob Agents 2017; 50: 507-511.

3. Bhat KS, Priya R, Krishnan L, Kanungo R. Elizabethkingia meningoseptica bacteremia in a neonate: A case report and mini-review of the literature. J Curr Res Sci Med 2016; 2: 42-5.

4. Masrani KM, Salunke PP, Ahmed J, Kabra NS. Elizabethkingia meningoseptica - an emerging cause of neonatal meningitis. Perinatology 2019; 19: 139-142.
5. Dziuban EJ, Franks JL, So M, Peacock G, Blaney DD. Elizabethkingia in children: A comprehensive review of symptomatic cases reported from 1944-2017. Clin Infect Dis 2018; 67: 144-149.

6. Chamalla SK, Karri SS, Koripadu S, Mohan N. Elizabethkingia meningoseptica: An emerging pathogen causing neonatal meningitis. Journal of Dr. NTR University of Health Sciences 2018; 7: 213-215.

7. Lau SK, Chow WN, Foo CH, Curreem SO, Lo GC, Teng JL, et al. Elizabethkingia anophelis bacteremia is associated with clinically significant infections and high mortality. Sci Rep 2016; 6: 26045.

8. Shinha T, Ahuja R. Bacteremia due to Elizabethkingia meningoseptica. IDCases 2015; 2: 13-15. 